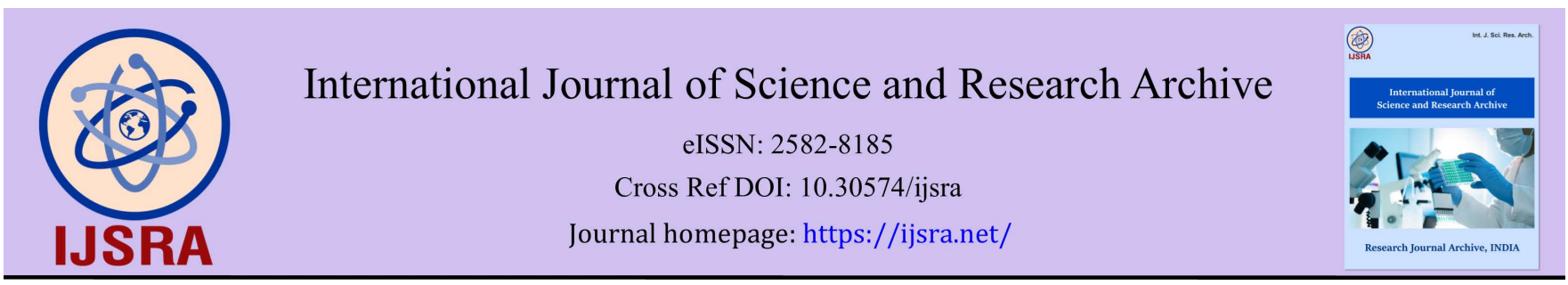

(REVIEW ARTICLE)

\title{
Anxiety management through hippotherapy in children with developmental disorders
}

\author{
Chrysoula Moscholouri ${ }^{1}$ and Konstantinos Chandolias 2,* \\ ${ }^{1}$ Department of Physical Therapy, University of West Attica, Greece. \\ 2 Department of Physical Therapy International Hellenic University, Greece.
}

International Journal of Science and Research Archive, 2021, 02(01), 106-110

Publication history: Received on 14 January 2021; revised on 10 February 2021; accepted on 12 February 2021

Article DOI: https://doi.org/10.30574/ijsra.2021.2.1.0031

\begin{abstract}
A large number of children with developmental disorders show symptoms of anxiety compared to children with normal development. High levels of stress weaken relationships with people around these children, thus limiting the social interaction they may have. In addition to having higher anxiety overall, children with developmental disorders also have a higher comorbidity of anxiety with other disorders. Hippotherapy is an intervention, which uses the natural gait and movement of a horse to provide motor, and sensory stimulation. It is a unique treatment strategy for children with disabilities, as it improves movement coordination and posture control. By improving movement, children with disabilities gain confidence and sense of security which helps them to deal with stress.
\end{abstract}

Keywords: Hippotherapy; Stress management; Children with developmental disorders

\section{Introduction}

Studies have shown that a large number of children with developmental disorders show symptoms of anxiety. Specifically, children with Autism Spectrum Disorder (ASD) have higher levels of anxiety symptoms than children with normal development and 40-50\% of these children meet the criteria for anxiety disorder. In addition, high levels of anxiety have been observed among children with Attention Deficit Hyperactivity Disorder (ADHD), Learning Disabilities (LD) and Intellectual Disabilities (IDs). Researchers have found that children with ASD, in particular, had high levels of anxiety [1].

High levels of stress interfere with daily activities and stress itself affects children with developmental disorders. In addition, high levels of stress weaken relationships with the people around the, friends, family and teachers, thus limiting the social interaction they may have. Also, while it has been suggested that there is no direct relationship, high levels of stress lead to limited and repetitive behaviors and intense persistence in routine. Restricting social interaction is believed to reduce learning opportunities for appropriate social skills, as without appropriate interactions it is less likely to acquire appropriate social skills and modify inappropriate social skills. In addition, high levels of stress lead to reduced ability to read social cues in interpersonal relationships, so it is difficult for these children to acquire appropriate social skills even if there is an opportunity for social interaction [2].

Incorporating animals into the treatment process to reduce problem behaviors and improve function has been suggested as a promising area of intervention for those populations at risk for anxiety disorders. Data on animal-assisted therapy (AAI) interventions are limited. However, the inclusion of animals in healthcare settings has been suggested to produce relaxation experiences related to senses that allow children to manage better stressful events and engage in social behaviors [3]. Studies have reported reductions in stress hormone levels (ie cortisol) in children with ASD after

\footnotetext{
${ }^{*}$ Corresponding author: Konstantinos Chandolias

Department of Physical Therapy International Hellenic University, Greece.
} 
interactions with dogs, and in a general pediatric population after intervention with 11-week-old horses. The ASD population has a particular need for interventions aimed at reducing negative stress-related behaviors and improving social interactions [4].

ASD is characterized by dysfunctional behaviors, communication difficulties and social interaction. Affected individuals usually exhibit stereotypical behaviors and struggle with establishing contact, verbal and non-verbal communication, creating and understanding interpersonal relationships. The etiology of ASD remains to be fully elucidated and the observation method is the only diagnostic tool [5]. The multifactorial nature of ASD is the reason why a variety of treatments, including educational, behavioral and pharmacotherapy, have been applied. Despite extensive research, there is no consensus on the effective management and pharmacotherapy of ASD. The available treatments are not enough even in terms of symptomatic treatment. Parents of children with ASD often seek complementary and alternative therapies, e.g. equine-assisted activities and therapies (EAAT), to support the classical approach. As the number of people undergoing EAAT increases annually, it seems prudent to evaluate the effectiveness of EAAT in treating ASD [6].

EAAT incorporates equine therapy (HPOT), a comprehensive treatment program, and therapeutic riding (TR), which comes from recreational activities. Both types of therapy use contact with horse and equine movement, which provides rhythmic movement in the patient's body. EAAT affects the whole body, regulating blood pressure and muscle tone and leads to improved balance. The literature offers many reports on the effectiveness of EAAT in improving bodily functions, although it is mainly for patients with cerebral palsy (CP)). However, the popularity of EAAT is increasing and the number of publications is increasing accordingly [7]

\section{Method}

A research was conducted for randomized control trials about the anxiety management through horse therapy in children with developmental disorders. Searched Medline databases (via Pubmed), PEDro (Physiotherapy Evidence Datadase) and Google Scholar. The terms used were hippotherapy, stress management, children with developmental disorders, with articles during the period 2000-2020.

\subsection{Anxiety Disorder in Children with Developmental Disorders}

Anxiety disorder has been repeatedly found to be associated with learning disabilities and also with autism. In addition, it is suggested that the comorbidity of autism and learning disabilities leads to a higher risk of psychiatric disorders. Extensive research has been done on the comorbidity of learning disabilities and autism. Researchers have concluded that adolescents with autism and severe learning disabilities are more vulnerable to anxiety disorders. The comorbidity especially of autism can also make children and young people with learning disabilities and autism more susceptible to anxiety disorders [8].

In addition to having higher overall anxiety, children with Intellectual Disability (ID) also have a higher coexistence of anxiety and other disorders. Numerous studies have found that twice as many children with ID and an anxiety disorder also met criteria for one or more co-occurring behavioral disorders (42.5\%, ie ADHD, ODD Oppositional Defiant Disorder), compared with children with typical growth and with anxiety disorders (20.6 - 20.9\%). This higher coexistence is of particular clinical importance, given the high level of attenuation observed in both groups of disorders and the possible interactions between extroversion symptoms and anxiety [9].

Certainly, it has been established that stress is associated with behavioral problems in both developmentally retarded and typically developed preschool children. Preschoolers who have been identified as developmentally retarded also show more problem behaviors and greater social skills deficits than their typically developing peers, resulting in less interactive and lonely play. In these children, stress management is an imperative as it determines the interactions with their environment [10].

\section{Hippotherapy and Indications for its Effectiveness}

As mentioned, hippotherapy is the intervention that uses the natural gait and movement of a horse to provide motor and sensory stimulation. It is based on the improvement of neurological functions, and sensory processes, and is used for patients with physical and mental disorders. Especially in people with paralysis it contributes to physical rehabilitation programs. Horse therapy means treatment with the help of a horse. Despite its long history of use, only a few studies have been carried out on its theoretical basis. Only a little information is available about its psychological, physical, social and educational effects on children with disabilities. Hippotherapy is a method of treatment with at the 
same time favorable psychological, social and educational effects on the sensory system, the musculoskeletal system and the visual systems [11].

This is a rapidly growing field for people with disabilities involving horses in a therapeutic intervention. Hippotherapy (hippotherapy - HPOT), psychoeducational horseback riding (PER). Although each method offers a different therapeutic focus and type of activity, including horses as part of the therapeutic experience is a common thread. THR usually involves small group riding led by a certified THR trainer who teaches riding skills aimed at therapeutic goals [4].

HPOT is a unique treatment strategy for children with disabilities, because it takes into account the treatment context while providing the support needed to challenge the cognitive-sensory systems. Active participation in therapeutic activities has been shown to lead to improvements in adjustment and increased willingness to participate in daily activities, and the use of horse movement as a therapeutic tool is theoretical to have similar effects. Children with ASD increased posture stability (regardless of age) compared with typically developing children during a permanent balance work measured with a power plate and kinematic data, supporting the hypothesis that growth retardation in children with ASD. Standing instability can affect fine motor skills, handling skills, and the design and execution of motor coordination. Views on the potential impact of impaired movement control on occupational therapy vary. However, standing control is related to language, social commitment, play skills and academic ability [12].

Also, depression and anxiety disorders in children and adolescents, even those of normal development, are a substantial and persistent public health problem that can destabilize social and school functioning, create family stress and require significant use of mental health services. While existing treatments may offer benefits, many clients give up or do not respond to treatment. Researchers believe that most children and adolescents do not receive the mental health treatment they need, with more than $70 \%$ of adolescents not receiving appropriate treatment. This is due to lack of resources, stigma associated with mental illness and inadequately trained health personnel, as well as misdiagnosis and inadequate mental health insurance [13].

Including an animal in treatment process is considered beneficial as animals seem to have a natural tendency to bond with humans. As an alternative to "therapist speech therapy", animal therapies are believed to go beyond some of the limitations of traditional counseling therapy. Many traditional therapies rely solely on language as a means of change, which may not be the most effective way to facilitate change in all clients, especially adolescents. The involvement of a horse as a means of healing offers many unique properties that are not accessible through other forms of healing. During treatment, horses are used as altering agents to enable growth, learning and development processes within the client. It has been suggested that the presence of the horse offers both emotional and physical comfort and therefore the development of trust and security, something that the therapist is not always able to offer [14].

Decreased self-confidence and self-esteem are primary characteristics of adolescent depression and anxiety and are the target of traditional interventions for adolescents with depression. The ability to control a large and intimidating animal and its movement without the help of others is considered to be an integral step in the process of increasing self-esteem and self-confidence. This makes it an attractive alternative or adjunct to current proven therapeutic treatments, as it directly affects the key components of anxiety and depression. EAAT provides a unique environment that facilitates change within adolescents experiencing depression or anxiety. As a result of horses' natural responses, adolescents are able to recognize "how their behaviors affect horses" and these results are obtained immediately. Participants can see the patterns of cause and effect in action that create useful and unhelpful interactions [15].

\section{Stress Management Through Hippotherapy in Children with Developmental Disorders}

The results of the study by Gabriels et al (2015), create hypotheses about the role of human-equine interaction that requires further research. One hypothesis is that riding and working with the horse to engage the child in therapeutic riding activities involves a non-verbal experience of shared or shared attention that can serve as a platform for improving children's social behaviors and communication skills. with ASD. This non-verbal communication between the horse and the rider may involve the fact that the horses are constantly reflecting and responding to the rider's body language. Also, this shared attention experience can be enhanced by the size of the horse combined with the rider's demand for movement to maintain bilateral control and balance. Common attention skills outcome measurements, including semi-structured play assessments and behavioral observation coding, may be useful estimates for future EAAT studies. A second hypothesis is that the human-equine experience (i.e., the warmth of the horse body and the rhythmic movement of the horse) promotes a relaxing framework, which may have a calming effect in children with ASD. The impact of EAAT on reducing stress levels can be investigated using objective behavioral observation measures in combination with biological measures such as galvanic skin response or salivary cortisol [4]. 
On the subject of the autism group, the development of motor function through hippotherapy is very important in autism and can have a direct impact on autonomy, suggesting the need for more work to also affect the cognitive, emotional and social aspects. According to this, hippotherapy can improve social relationships because it favors a better perception of the outside world and its impact on tonic-orthostatic adjustments. There seem to be similarities in the autistic person's behavior and some horse postures. Loud noises, changes in the familiar and unfamiliar environment can cause insecurity in both cases, and much of the communication is based on body language. In addition, the horse's instinctive ability to perceive the rider's intentions relaxes the animal when guided by a person with autism[16].

These improvements in posture control have been found in some of the specific populations mentioned and serve to reiterate the idea that this treatment is beneficial for these individuals. Along with improvements in controlling movement and posture, equine research also suggests that social and emotional interactions can lead to increased quality of life and self-esteem. Thus, this rehabilitation strategy is ideal for populations that will benefit from strengthening control of their upright posture, as well as for building social interactions in an unconventional way. Musculoskeletal system coordination is an integral part of balance, mobility and independence. In addition, coordination and control difficulties can affect the psychological and cognitive development of individuals. Therapeutic interventions, such as hippotherapy, are needed in order to enhance normal growth and restore recovery, improve coordination and quality of life. Motion scientists are particularly interested in controlling the movement and posture of children in order to address the coordination issues that play a role in the quality of life of individuals. A specific population, people with cerebral palsy, has been shown to have consistently positive motor effects after hippotherapy interventions. Cerebral palsy, that is, delayed or impaired brain development, consists of a number of disorders, including disabilities in the development of movement as well as posture. Patients receiving hippotherapy usually show improvements in motor results because a horse's gait is very similar to that of a normal human walking pattern. Therefore, a person who cannot walk independently is able to assimilate this pattern through the horse's gait [17].

\section{Conclusion}

Children and adolescents with developmental disorders are at increased risk of developing anxiety disorders, at a higher rate than children with normal developmental disorders. Stress management in these children is essential, as it is a prerequisite for enhancing their social interactions, and thus their socialization. The inclusion of animals in therapeutic regimens has been suggested to produce relaxation experiences related to the senses that allow children to better manage stressful events and engage in social behaviors. Even in populations of typically developing adolescents and children, contact with animals has been shown to prevent feelings of anxiety and depression.

Hippotherapy has been established as an effective treatment for children with a variety of developmental disorders. These results are probably due to the constant movement of the horse, forcing participants to steadily correct their torso positions to stay upright. The use and involvement of the torso muscles ultimately strengthens them, allowing better posture control after treatment. Improvements in walking coordination give children with developmental disorders confidence and a sense of security. In addition, especially for children with autism spectrum disorder, who usually experience severe anxiety disorders, hippotherapy has been shown to be effective in both movement coordination and stress management. The above findings advocate the use of hippotherapy for the management of stress and all comorbidities in all children with developmental disorders.

\section{Compliance with ethical standards}

\section{Acknowledgments}

The authors would like to acknowledge International Hellenic University and the MSc program Pediatric Physiscal Therapy for the opportunity to conduct this review.

\section{Disclosure of conflict of interest}

The authors report no conflict of interest.

\section{References}

[1] Ishimoto Y, Yamane T, Matsumoto, Y. Anxiety Levels of Children with Developmental Disorders in Japan: Based on Reports Provided by Parents, Journal of Autism and Developmental Disorders. 2019; 49(9): 3898-3905. 
[2] Kleinhans NM, Richards T, Weaver K, Johnson LC, Greenson J, Dawson G, Aylward E. Association between amygdala response to emotional faces and social anxiety in autism spectrum disorders, Neuropsychologia. 2010; 48(12): 3665-70.

[3] Johnson RA. «Animal-assisted intervention in health care contexts,» $\sigma \varepsilon$ How Animals Affect Us: Examining the Influence of Human-Animal Interaction on Child Development and Human Health, P. McCardle, S. McCune , J. A.

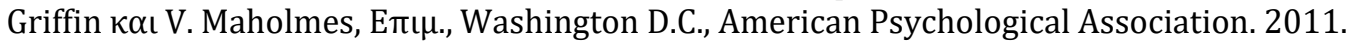

[4] Gabriels RL, Pan Z, Dechant B, Agnew JA, Brim N, Mesibov G. Randomized Controlled Trial of Therapeutic Horseback Riding in Children and Adolescents With Autism Spectrum Disorder, Journal of the American Academy of Child and Adolescent Psychiatry. 2015; 54(7): 541-549.

[5] Saghazadeh A, Ahangari N, Hendi K, Saleh F, Rezaei N. Status of essential elements in autism spectrum disorder: systematic review and meta-analysis, Reviews in the Neurosciences. 2017; 28(7): 783-809.

[6] Trzmiel T, Purandare B, Michalak M, Zasadzka E, Pawlaczyk M. Equine assisted activities and therapies in children with autism spectrum disorder: A systematic review and a meta-analysis, Complementary Therapies in Medicine. 2019; 42: 104-113.

[7] Whalen CN, Case-Smith J. Therapeutic effects of horseback riding therapy on gross motor function in children with cerebral palsy: a systematic review, Physical \& Occupational Therapy in Pediatrics. 2012; 32(3): $229-42$.

[8] Hajizadehanari K, Hajizadehanari H, Yaghoubzadeh A, Dalir A, Farhadian A, Askari M, Hosseini Fard SMHS. Anxiety disorders in children with learning disabilities (LD) and autism (ASD), The European Journal of Social \& Behavioural Sciences. 2013; 5(2): 937-945.

[9] Green SA, Berkovits LD, Baker BL. Symptoms and development of anxiety in children with or without intellectual disability, Journal of clinical child and adolescent psychology. 2015; 44(1): 137-144.

[10] Crnic K, Hoffnan C, Gase C, Edelbrock C. Understanding the Emergence of Behavior Problems in Young Children With Developmental Delays, Infants and Young Children. 2004; 17(3): 223-235.

[11] Koca TT, Ataseven H. What is hippotherapy? The indications and effectiveness of hippotherapy, North Clin Istanbul. 2016; 2(3): 247-252.

[12] Ajzenman HF, Standeven JW, Shurtleff TL. Effect of Hippotherapy on Motor Control, AdaptiveBehaviors, and Participation in Children WithAutism Spectrum Disorder: A Pilot Study, The American Journal of Occupational Therapy. 2013; 67(6): 653-63.

[13] Wilson K, Buultjens M, Monfries M, Karimi L. Equine-Assisted Psychotherapy for adolescents experiencing depression and/or anxiety: A therapists perspective, Clinical Child Psychology and Psychiatry. 2015; 22(1).

[14] Selby A, Smith-Osborne A. A systematic review of effectiveness of complementary and adjunct therapies and interventions involving equines, Health Psychology. 2013; 32(4): 48-32.

[15] Notgrass CG, Pettinelli DJ. Equine Assisted Psychotherapy: The Equine Assisted Growth and Learning Association's Model Overview of Equine-Based Modalities, Journal of Experiential Education. 2015; 38(2).

[16] Portela-Pino I, Bouzo-Gonzalez S, Pino-Juste M. Evaluation of an equine therapy program in students with Autism spectrum disorder, Journal of Human Sport \& Exercise. 2020; 15(4).

[17] Thompson F, Ketcham CJ, Hall EE. Hippotherapy in Children with Developmental Delays: Physical Function and Psychological Benefits, Advances in Physical Education. 2014; 04(02): 60-69. 